\title{
DENTAL IMPLANTATION IN PATIENTS WITH DIABETES AND DIFFUSIVE TIREOTOXIC GOITER WITH ACCOUNT OF EVALUATION OF MICROCIRCULATION IN VESSELS
}

\author{
Kudratov Shuxrat Sheraliyevich, \\ Master of Medical sciences Tashkent State \\ Dental Institute \\ Khassanov Sardor Mukhitdinovich, \\ master student Tashkent State Dental Institute \\ 2 course email sardor.khasanov2016@yandex.ru \\ Feofanidi Yuliya Iordanovna, \\ student Tashkent State Dental Institute 4 course
} http://dx.doi.org/10.26739/2573-5616-2018-3-2-8

\begin{abstract}
Terms of effective use of dental implants in depend on the biological processes of interaction of the implant and surrounding tissues, including the functional state and reactive properties of soft tissues in the implantation area. Clinical and experimental studies of periodontium and organization of regional hemodynamics indicate a key role microcirculation in the formation and management of adaptive compensatory processes. As is known, in diabetes mellitus there is a persistent violation of the microcirculation of blood vessels, depending on the timing of the disease. In addition, the view is growing that hyperthyroidism leads to a violation of blood circulation in small vessels.
\end{abstract}

Key words: Key words. Dental impantation, diffuse thyrotoxic goiter, diabet 2 type, hyperthyreose, ossteointegration, microcirculation, peri-implantitis.

\section{Description.}

Dental implantation is one of the priority tasks of modern dentistry. The use of implants as artificial dental supports allows solving numerous problems of prosthetics of patients with partial and complete absence of teeth, thereby improving the quality of life of patients. At present, the question of osseointegration of dental intraosseous implants in patients with diabetes mellitus and hyperthyroidism is of the greatest scientific and practical interest.

Diabetes mellitus and hyperthyroidism are one of the most important problems of modern clinical endocrinology, due to their prevalence and the development of changes on the part of all organs and systems. Therefore, researchers pay great attention to the study of these pathologies. 
Despite significant progress in the diagnosis and pathogenesis of diabetes mellitus and hyperthyroidism, in studying their effect on various organs and systems of the body, many aspects of problems remain insufficiently illuminated, in particular, the state of the microvasculature in the vessels. Changes in microcirculation in diabetes mellitus and hyperthyroidism are manifested by moderate signs of osteoporosis, as well as the quality of osseointegration in dental implantation.

Diabetes mellitus (DM) is a group of metabolic (metabolic) diseases characterized by chronic hyperglycemia, which is the result of impaired insulin secretion, insulin action, or both. [1]. According to the International Diabetes Federation (IDF), in 2015 there were 415 million patients with DM in the world (one in eleven adults). By 2040 IDF predicts an increase in the number of patients to 642 million (one in ten adults) [2].

The development of complications of diabetes mellitus is accompanied by systemic disorders of blood microcirculation. Morphological and functional changes in the vessels of the microcirculatory flow along with rheological disorders are observed even in early (including preclinical) stages of complications development (3). Early microcirculatory disorders are recognized as a promising clinical marker of progressive complications of diabetes mellitus (4). The degree of disturbance of microcirculation is often related in geometric progression with the period of the development of the disease.

Determination of microcirculation in small vessels is one of the key factors in the planning and prevention of complications after the operation of dental implantation in patients with diabetes.

Similarly, the structure of the endocrine pathology is one of the leading places for thyroid diseases. Diffuse toxic goiter (DTG) - is one of the most common diseases of the thyroid gland and the most clinically significant thyroid pathology, which occurs in $0.5-1.5 \%$ of the population. It is established that in the regions of iodine deficiency, to which the Republic of Uzbekistan belongs, the prevalence of DTG is higher in comparison with iodine-filled regions. It should be noted that at present there is an increase in morbidity and an increase in the incidence of severe forms of pathology. Hyperthyroidism causes an increase in osteoblastic function, which is expressed by an increase in the amount of osteocalcin in the blood and an increase in the activity of alkaline phosphatase. However, increased bone formation does not compensate for sharply increased bone resorption, which subsequently leads to a decrease in bone mineral density.

Disease of diabetes and DTG due to circulatory disturbances to varying degrees, as well as the violation of calcium metabolism in the body leads to osteoporosis. Along with other disorders in patients with a history of 
diabetes and DTG dental implantation has certain features associated with the preparation and introduction of these patients with endocrine pathologies.

Purpose of the study: Morphofunctional evaluation and study of the results of dental implantation in patients with diabetes mellitus and diffuse thyrotoxic goiter, taking into account the evaluation of microcirculation in the blood vessels

Materials and methods: For the operation of dental implantation, 30 patients with a history of type 2 diabetes and 30 patients with a previous history of DTG were selected. As a control group, 30 patients without a history of diabetes mellitus were selected. The average age of the patients was 34,3 ë 8, 5 years. Sixteen patients underwent implantation in the region of the front teeth, 33 in the region of the lateral teeth, and 11 patients underwent implantation in the frontal and lateral regions. Often in these diseases, there are various periodontal diseases, as well as the accumulation of soft and hard dental deposits in the oral cavity. Patients with unsatisfactory hygienic condition of the oral cavity were prescribed preoperative professional cleaning of the oral cavity, rinsing with the drug "Loroben".

For the evaluation and planning of the operation, radiation diagnosis of the maxillofacial region was carried out before and after dental implantation, and also after a week, a month, three months and a half a year.

Violations of the microcirculation of blood vessels can often be detected on the chamber. Due to the availability for visual analysis, the microcirculatory bed of the retina, compared with that of other organs, is best studied. Thus, examination the eye chamber by ophthalmologist routine procedure analyzing patients with diabetes mellitus. To assess the microcirculatory flow, we conducted with the eye clinic in Tashkent, where the method of assessing the retinal picture was used by the "OCT Spectralis" company of HEILDERBERG ENGINEERING (Germany).

The study was conducted before and after preoperative specialized therapy at 1 month prior to surgery, 1 week after surgery, 1 month and 3 months after surgery. Patients for

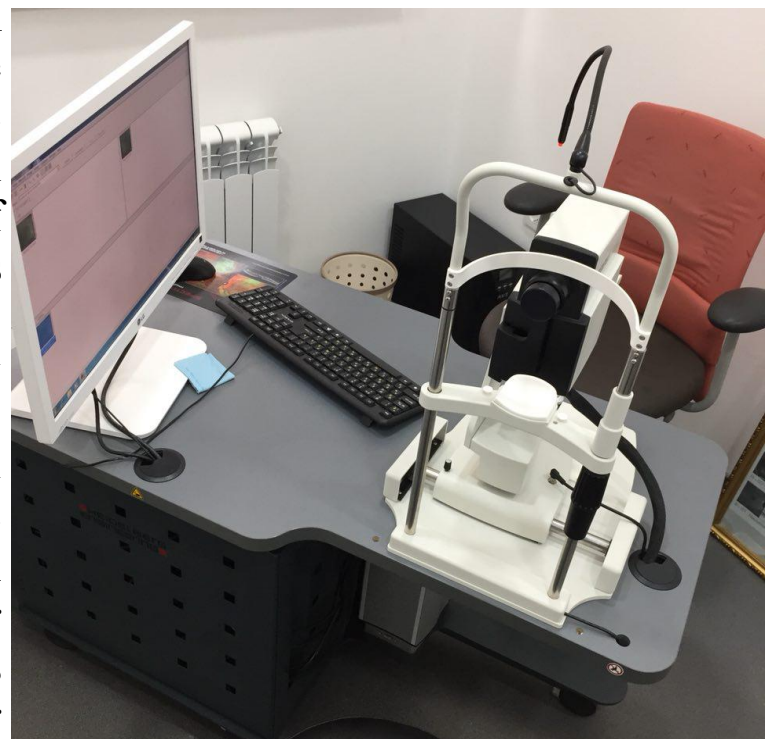


correction of blood circulation by endocrinologists were assigned various drugs for conservative therapy (antiaggregants, anticoagulants, statins, etc.)

After the atraumatic incision and detachment of the mucous-periosteal flap, a bone bed was formed for the dental implant using the generally accepted principles of atraumatic bone preparation. The bed was prepared in stages with the gradation of instruments by diameter and constant irrigation of the zone of operation with a cooling solution. The implant was installed in accordance with the shape and size of the bone bed. Closure of the surgical wound was performed by applying nodular or continuous sutures.

The criteria for assessing the primary healing of a postoperative wound after dental implantation were local and general manifestations of various traits. To the local we included: pain, edema, redness, the condition of the stitches. Common signs include signs of intoxication headache, malaise.

All patients in the dynamic for objective evaluation of early diagnosis and distant Osseointegration were measured osseointegration of dental implants by frequency resonance analysis with the Osstell ISQ MEGAGEN (Implant Stability Quotient) device .The studies were performed during the operation on an open wound, after surgery, at 1, 2, 4, 6, 9, 12 and 24 months, these periods in groups 1-3 often coincided during the period of prosthesis and functioning of the prosthesis. The measurement of ISQ / CSI during the operation played a determining role to assess the primary stability on which the preliminary period of the second stage of surgical treatment depends - the settings of the superstructure or the beginning of the functional load of the implant with the help of prosthesis.

Results of the study: The evaluation criteria for the result of the cone ray computed tomography were: evaluation of the position of the dental implant relative to anatomical formations (maxillary sinus, mandibular canal, and chin aperture), the structure and relative density of bone tissue surrounding the dental implant. The presence of the enlightenment zone was regarded as an unfavorable result of the osseointegration of the dental implant. The structure of the shadow of bone tissue was assessed as nonuniform, areas of dense bone tissue alternated with areas of bone enlightenment - which is the optimal result. Density of tissues was estimated by Hounsfield in figures (HU CT number, "Hounsfield Unit"). The average rate for Hounsfield before the operation began was 600 to 800 units, after surgery, the indices were 550-700 units, a month after operation 650- 750 units.

At DTG disturbances of microcirculation in vessels are not observed in the majority of patients, however, in some patients, various degrees of expansion of blood vessels were revealed. 

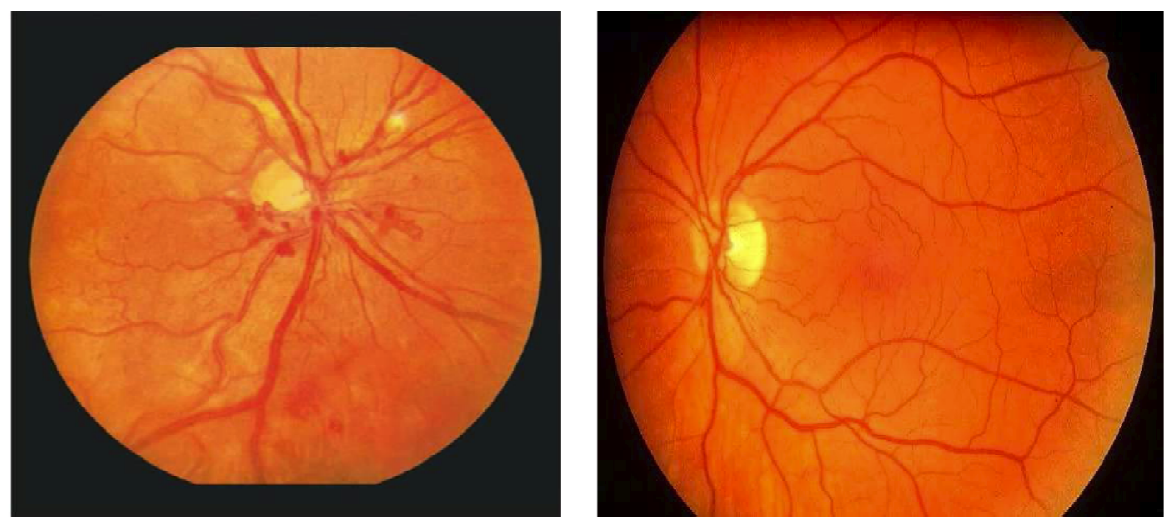

Table1. Results of changes in the microcirculation of the vessels on the chamber

\begin{tabular}{|c|l|}
\hline Diagnosis & $\begin{array}{c}\text { Diabetes type 2 } \\
\text { Number of patients }\end{array}$ \\
\hline $\begin{array}{c}\text { Non-pluritransitive } \\
\text { Retinopathy (Diabetic retinopathy 1) }\end{array}$ & 5 \\
\hline Pre-proliferative retinopathy (diabetic retinopathy 2) & 1 \\
\hline Proliferative retinopathy (diabetic retinopathy 3) & 2 \\
\hline Extension of blood vessels & 5 \\
\hline
\end{tabular}

We estimated the wound healing time using evaluation criteria for the area of the postoperative site where dental implantation was performed.

\begin{tabular}{|l|c|c|c|c|}
\hline $\begin{array}{l}\text { Evaluation } \\
\text { criteria }\end{array}$ & After 1 day & After 3 days & After 5 days & After a week \\
\hline Pain & + & + & - & - \\
\hline Edcma & + & ++ & - & - \\
\hline Redness & + & ++ & - & - \\
\hline Temperature & + & + & - & - \\
\hline Highlighted & - & - & - & - \\
\hline Weakness & - & + & - & - \\
\hline
\end{tabular}

Note: + intensively ++ significantly

- Unimportant or absent 
The optimal ISQ / CSI index for a safe functional load is above 70 units. When assessing osseointegration, the accuracy of measurements of the stability of implants, the Osstell instrument (Sweden), with sufficiently rigid attachment of parts, repeatability was established with high accuracy.

In patients without a history of endocrine disorders, the ISQ / CSI index during the implantation had a mean of $76( \pm 2.5)$, after 1 month the ISQ / CSI remained within $80( \pm 1.0)$ and in terms of 2-4-6 -9-12 months ISQ / CSI indicators with a tendency to increase from $80( \pm 1.0)$ to $88( \pm 1.0)$. After 24 months the ISQ / CSI remains stable unchanged.

In patients with a history of diabetes mellitus type 2, the average ISQ / CSI during implantation $68( \pm 1.5)$, after 1 month $71( \pm 1.0)$ and in terms of 2-4-6-9-12 months, the indices ISQ / CSI with a tendency to increase from $73( \pm 1.0)$ to $80( \pm 1.0)$. After 24 months, the ISQ / CSI are increased to $81( \pm 1.5)$.

In patients with the feature of DTG the average ISQ / CSI value during implantation of was $68( \pm 1.5)$, after 1 month $75( \pm 1.5)$ and in terms of 2-4-6-9-12 months, ISQ / CSI with a tendency to increase from $78( \pm 1.0)$ to $80( \pm 1.5)$. After 24 months, the ISQ / CSI are increased to $85( \pm 0.5)$.

All patients traditionally have implants with the same shallow screws of the intra osseous part.

\section{Conclusion}

Radiation diagnosis of patients before and after dental implantation allowed us to plan further operations, and also helped us evaluate the quality of the bone around the dental implant.

Based on the results of the analysis of the bottom of the eye chamber, osteopenia syndrome and violation of microcirculation in vessels in patients with type 2 diabetes mellitus were revealed. The degree of impairment ranged from vasodilatation to diabetic retinopathy with grade 3 .

Preoperative correction of hormonal imbalance, the appointment of targeted drugs for correction of blood circulation, improvement of reparative regeneration allowed us to prevent complications associated with the formation and fusion of bones with a dental implant.

Using the "Ostell ISQ" device (Resonance Frequency Analysis) to assess the coefficient of primary stability of the implant and distant osseointegration, allows us to prevent complications associated with unreasonable early loading of the implant and the selection of the optimal term for prosthetics, as well as reducing the duration of orthopedic treatment. 


\section{Literature:}

1. Smirnov A.N. Elements of endocrine regulation. - Moscow: GEOTAR-Media, 2006 - 352 pp. [SmirnovAN. Elementy endokrinnoy regulyatsii. Moscow: GEOTAR-Media; 2006352 p. (in Russ.)]

2. IDF Diabetes Atlas, 7th edn. Brussels, Belgium: International Diabetes Federation; 2015 Available from: http://www.diabetesatlas.org.

3. Koscielny J, Latza R, Wolf S, Kiesewetter H, Jung F.Early rheological and microcirculatory changes in children with type I diabetes mellitus. Clin Hemorheol Microcirc. 1998;19(2):139- 50.

4. Greenman RL, Panasyuk S, Wang X, Lyons TE,Dinh T, Longoria L, Giurini JM, Freeman J, Khaodhiar L, Veves A. Early changes in the skin microcirculation and muscle metabolism of the diabetic foot. Lancet. 2005;366(9498):1711-7.doi:http://dx.doi.org/ 10.1016/S0140-6736(05)67696-9. 